points of fusion of Sertoli cells showed particles in linear arrays on the $A$ face with corresponding pits on the $B$ face. This unique, occluding junction would be an effective barrier, protective against substances in circulation. This barrier, suggested Dr Fawcett, could perhaps be opened up to allow the penetration of a contraceptive substance.

Dr P. L. Pearson (State University of Leiden) discussed the manipulation of the action of the $Y$ chromosome genes and the production of an abnormal gamete as a possible method of contraception. Of the three phases of DNA synthesis (S phases), those occurring at preleptene and zygotene in the meiotic cycle have normal repair replication patterns and any interruption of synthesis here would be unspecific for any meiotic cell. At the third $S$ phase, at pachytene, there is abnormal repair, however, and if a particular endonuclease is featured here, then a block at this stage would be specific for spermatogenesis. Although most of this research has been carried out in plants (Lilium $\mathrm{sp}$.), there is evidence that similar repair mechanisms occur in mammalian cells.

Spermatogenesis was also the target of Dr D. Lacy (St Bartholomew's Medical School, London), who discussed the possibility of reducing the testicular weight (and thus spermatogenesis) while maintaining or stimulating the seminal vesicles using testosterone or a synthetic androgen. This differential response could perhaps be achieved by using a centrally acting oestrogen to prevent local production of steroids, followed by androgen administration to stimulate the seminal vesicles to take up testosterone. Work in his laboratory with rats suggests that this differential response might also be achieved in man.

Dr J. M. Bedford (Cornell University Medical College) suggested contraception by a chemical change mediated through the sperm cell surface which would affect its ability to fertilize the ovum. His study on the maturation changes as sperm pass through the epididymis concentrated chiefly on four aspects--the acrosome, the surface membrane, the nucleus and motility. Acrosome morphology was unchanged, but he found significant changes in the cell surface, the state of which is critical for the ability to fertilize. The nucleus also showed changes in the degree of covalent cross-linking. The available sulphydryl groups provided by the cysteine of the nuclear protein become cross-linked and confer greater structural rigidity on the mature sperm. The capacity for progressive movement rather than weak tail thrashing is accompanied by changes in the quality of the tail fibres during maturation; modifications to render sperm immobile would be difficult, but Dr Bedford suggested that they may be worth considering. Changes in nuclear development, however, are of functional importance in embryonic development and it was argued by Dr R. A. Beatty (University of Edinburgh) that the nonnuclear change affecting, for example, the accessory glands was far preferable to damage of genetic information and possible mutagenicity.

There was a considerable body of opinion that the epididymis is the site for contraceptive action. Dr D. Hamilton (Harvard Medical School) discussed its physiology as related to anatomical structure and showed that interference with any constituent of the lumen resulted in impairment of the functional integrity of the epithelium.

Dr G. Waites (University of Reading) described the passage of substances from the blood into the testicular lymph and fluid. All pass freely into the lymph but can be divided into three groups depending on their rate of entry into the testicular fluid, which does not depend upon molecular weight. A steroid could therefore be altered to prevent its diffusion but still act systematically as an androgen. Dr Waites suggested that this could be a useful screening technique, and the epididymal sterilant could be designed to act upon the rete testis. He warned, however, that, because of the similarity of the testicular fluid and brain fluids, the "ideal" fluid might also enter the blood/brain barrier; cerebrospinal fluid should therefore be included in the screen.

The technique described by Dr Waites for continually collecting large quantities of fluid for a long time without contact with the accessory organs greatly aided the study of metabolism and survival of sperm in the epididymis by $\mathrm{Dr}$ I. G. White (University of Sydney). $\mathrm{He}$ has found that testicular sperm metabolizes and incorporates glucose in the form of inositol, suggesting that this may be a reserve form of carbohydrate for sperm passage through the tract. The high concentration of phospholipid in testicular sperm becomes reduced as they pass along the epididymis, but the lipid does not appear in the plasma and Dr White suggested there may well be a sodium and potassium pump to maintain cation gradients. This, he felt, could perhaps be exploited in the development of a contraceptive.

The characterization of an acrosomal enzyme, acrosin, was described by $\mathrm{Dr}$ H. Fritz (University of Munich). Active acrosin is required for penetration; artificial inactivation either by interfering with its natural inhibitor or by permanently inactivating the enzyme could lead to new contraceptive ideas.

The place of the routine screen for a male contraceptive was discussed by $\mathrm{Dr}$ M. M. Ketchel (Oak Ridge Population Research Institute), who pointed out that it should be possible to develop compounds without side effects by either utilizing enzymes affecting the

\title{
Fluctuating Numbers of Ribosomal Genes
}

IT has been assumed that, with certain notable exceptions, the content of ribosomal RNA cistrons in each cell is a fixed number for any given genotype. Howell now reports in next Wednesday's Nature New Biology (December 27) that, in Chlamydomonas reinhardi, fluctuations in the genome content of rDNA occur during a single cell cycle.

When labelled rRNA was extracted from Chlamydomonas cells and run on an MAK column, two fractions were obtained-an $18 \mathrm{~S}$ species and a mixed fraction containing $18 \mathrm{~S}$ and $25 \mathrm{~S}$ rRNA. It was then calculated, from DNARNA hybridization experiments with these fractions, that the number of rRNA cistrons per cell in continuously growing cultures is 400 for $18 \mathrm{~S}$ rRNA and 250 for $25 \mathrm{~S}$ rRNA. Both $18 \mathrm{~S}$ and 25S rRNA hybridize to a DNA fraction of unique buoyant density.

In synchronized cells, however, the percentage of DNA which hybridizes at saturation to rRNA varies during the cell cycle. For both $18 \mathrm{~S}$ and $25 \mathrm{~S}$ species the amount of rDNA per cell rises sharply after cell division, then increases more gradually until the next division when the content of rDNA per cell falls abruptly. The difference in the values of the highest and lowest number of rDNA cistrons per cell during the cycle represents more than a three-fold change in the cellular content of both species of rDNA.

Howell correlates the pattern of rDNA degradation and synthesis with breakdown and reformation of the nucleolus during the cell cycle. $\mathrm{He}$ proposes a model relating rDNA metabolism with the nucleolar cycle. A limited number of rRNA cistrons, located at the nucleolar organizer, are considered to be an integral part of the chromosome. Additional nonchromosomal copies of the rRNA cistrons exist as part of the nucleolar structure. They are degraded during nucleolar breakdown and are not passed on to daughter cells. Following division and during nucleolar reformation additional non-chromosomal copies of rDNA are resynthesized.

According to the model the cellular content of rDNA at any point in the cell cycle will be the product of synthetic and degradative control of non-chromosomal rDNA and, in cells which have "persistent" nucleoli during mitosis, there is no degradation and re. synthesis of nucleolar rDNA. 